\title{
Postpartum acute MI following routine ergometrine administration treated successfully by primary PTCA
}

\author{
N Sutaria, L O’Toole, D Northridge
}

A 28 year old white woman had an unplanned home birth due to a short second stage of labour. A healthy boy was delivered vaginally with a midwife in attendance. Before delivering the placenta the patient was given $1 \mathrm{ml}$ of intramuscular Syntometrine (ergometrine maleate $500 \mu \mathrm{g}$, oxytocin 5 units; Novartis, Surrey, UK). The placenta and membranes were delivered completely with minimal blood loss. Fifteen minutes later the patient complained of severe central chest tightness radiating to both arms and associated with profuse sweating, nausea, and breathlessness. She was transferred to a local maternity hospital for further assessment.

On admission her ECG showed ST segment elevation across the chest leads with $6 \mathrm{~mm}$ of ST elevation in leads V4 and V5 confirming an acute anterior myocardial infarction (MI). She was transferred to the coronary care unit at a nearby district general hospital. Thrombolysis was withheld due to the risk of postpartum haemorrhagic complications and she was immediately transferred to a tertiary referral centre 25 miles away where she underwent emergency cardiac catheterisation.

The patient had been well previously with no cardiac symptoms and two entirely uncomplicated pregnancies. However, she was a heavy smoker and hypercholesterolaemia was diag-
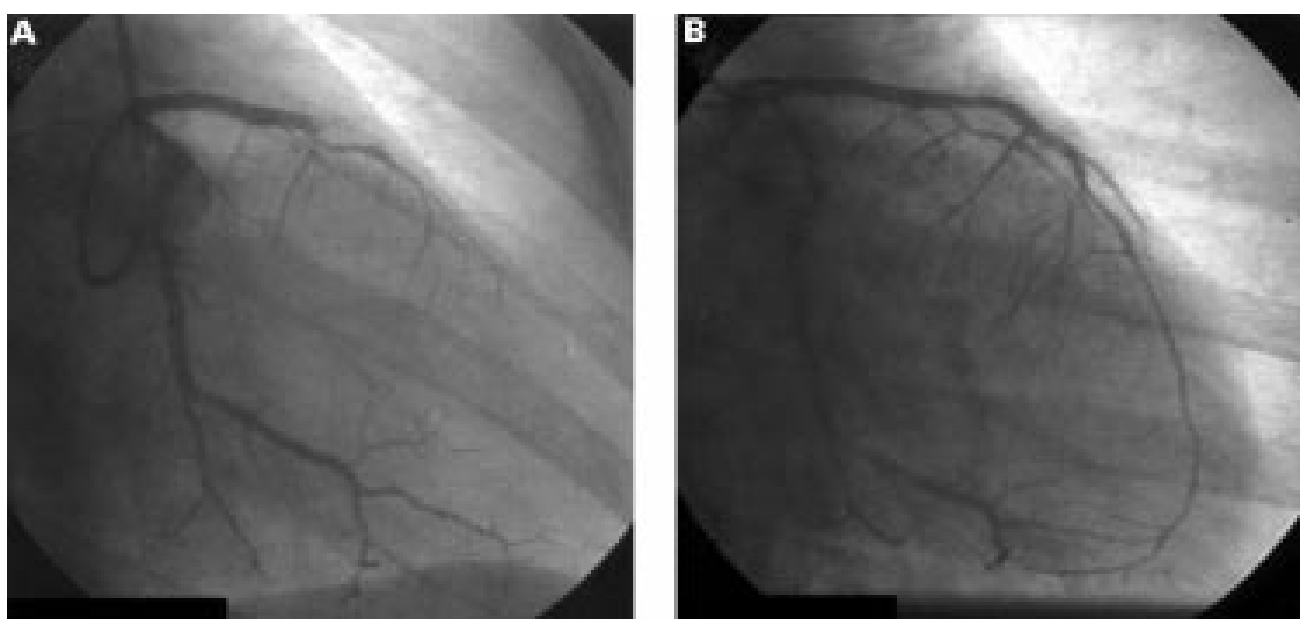

Figure 1 Left coronary angiograms showing $(A)$ acute left anterior descending coronary artery occlusion and (B) restoration of flow following PTCA and stent insertion.

nosed after both parents died from coronary artery disease in their early 40 s.

Coronary angiography revealed diffuse three vessel coronary artery disease with an acute occlusion in the proximal left anterior descending coronary artery (TIMI grade 0 flow) (fig 1A). The occlusion was dilated with a $3 \mathrm{~mm}$ balloon followed by insertion of a $9 \mathrm{~mm}$ NIR stent with a good angiographic result (fig 1B). TIMI grade 3 flow was restored to the infarct related artery within half an hour of arrival at the tertiary centre ( 5.5 hours after the onset of chest pain). A subsequent ECG showed resolution of ST elevation and Q waves in leads V1-4. Creatinine kinase (CK) peaked at $9858 \mathrm{U} / 1 \quad(8 \%$ CK-MB isoenzyme). Echocardiography showed anteroseptal hypokinesis but other segments of the left ventricle contracted well. Her recovery was uncomplicated.

\section{Discussion}

Acute MI occurs rarely in women of childbearing age and has a reported incidence in pregnancy of 1 in $10000 .{ }^{1} \mathrm{MI}$ is more frequent during the third trimester and puerperium of the first and second pregnancies and most commonly involves the left anterior descending artery territory. In the immediate postpartum period spontaneous coronary artery dissection is the most common cause of $\mathrm{MI} .^{2}$ In $20 \%$ of

Department of Cardiology, Western General Hospital, Crewe Road, Edinburgh EH4 2XU, UK

N Sutaria

L O'Toole

D Northridge

Correspondence to: Dr Sutaria.

Accepted for publication 7 January 1999 
cases of MI in pregnancy, thrombus is identified without angiographically visible atherosclerotic disease. A combination of transient coronary artery spasm and the hypercoagulable state of pregnancy may lead to coronary thrombosis.

In most maternity units Syntometrine is given routinely at the time of delivery of the placenta and membranes to reduce postpartum haemorrhage. Ergometrine is a potent vasoconstrictor, which is also used in provocation testing for coronary vasospasm. Refractory vasospasm culminating in $\mathrm{MI}$ is a recognised complication in this setting, and occlusive coronary artery spasm leading to in situ thrombosis has been shown angiographically. ${ }^{3}$ However, there have been only two previous reports of MI resulting from postpartum use of ergometrine. ${ }^{45}$ In both reports the patients had angiographically normal coronary arteries. In our report the patient had extensive coronary atherosclerosis, which may have enhanced the vasoconstrictor response to ergometrine.

Treatment of MI in pregnancy and the puerperium is complicated as thrombolytics are contraindicated. PTCA is a viable alternative, although there are only eight reported cases of PTCA in pregnancy and the puerperium. To our knowledge this is the first reported case of primary PTCA carried out immediately after delivery and only the second report of intracoronary stenting for acute $\mathrm{MI}$ in pregnancy.

1 Ginz B. Myocardial infarction in pregnancy. F Obstet Gynaecol Br Commun 1970;77:610.

2 Roth A, Elkayam U. Acute myocardial infarction associated with pregnancy. Ann Intern Med 1996;125:751-62.

3 Hays J, Hamill D, Defelice C, et al. Coronary artery spasm culminating in thrombosis following ergometrine stimulation. Cathet Cardiovas Diag 1993;28:221-4.

4 Taylor G, Cohen B. Ergometrine-induced coronary artery spasm and myocardial infarction after normal delivery. $\mathrm{Ob}$ stet Gynaecol 1985;66:821-2.

5 Liau J, Cockrill B, Yarchak P. Acute myocardial infarction after ergometrine administration for uterine bleeding. $A m \mathcal{F}$ Cardiol 1991;68:823-4.

\title{
SHORT CASES IN CARDIOLOGY
}

\section{Histiocytoid cardiomyopathy presenting with Wolff-Parkinson-White syndrome}

\author{
M D Cabana, O Becher, A Smith
}

\section{Department of \\ Pediatrics, Carnegie Building \#291, The Johns Hopkins Hospital, 600 North Wolfe Street, Baltimore, $M D$ 21287-6220, USA M D Cabana O Becher}

\section{Department of} Pathology, The Johns Hopkins University School of Medicine, Baltimore, Maryland, USA

A Smith

Correspondence to: Dr M D Cabana, Department of Pediatrics and Communicable Diseases, University of Michigan Health System, D3202 Medical Professional Building 0718, $1500 \mathrm{E}$ Medical Center Drive, Ann Arbor, MI 48019-0718, USA.

email: mcabana@umich.edu

Accepted for publication 17 March 1999
A 14 month old African American girl with a history of Wolff-Parkinson-White (WPW) syndrome presented to the emergency department apnoeic, cyanotic, and without a pulse. She had suddenly become limp and unarousable. She did not respond to resuscitative efforts and was pronounced dead.

The patient had been diagnosed with WPW when she was 6 months old, after an irregular rhythm was noted in a routine examination. ECG showed normal sinus rhythm with infrequent preventricular contractions, a delta wave, a PR interval of 0.06 seconds, and a QRS interval of 0.096 seconds. QTc and axis were normal. Echocardiography revealed a normal sized heart with no structural abnormalities. She was not treated for WPW because she did not have episodes of arrhythmia on 72 hour Holter monitoring.

Necropsy revealed congestion of the lungs with prominent lymphatic dilatation and cardiomegaly. The right ventricular wall was thin and two subendocardial nodules (fig 1) were identified near the atrioventricular valves. The left atrial nodule measured $0.2 \mathrm{~cm}$ and the right atrial nodule measured $0.5 \mathrm{~cm}$.

Microscopically, the myocardium and subendocardial muscle of both ventricles con- tained bundles of large round myocytes with smooth borders, granular and eosinophilic cytoplasm, and small slightly irregular nuclei (fig 1). Electron microscopy showed increased mitochondria and loss of myofibres in these histiocytoid cells, consistent with the diagnosis of histiocytoid cardiomyopathy.

Histiocytoid cardiomyopathy is a rare myocardial disorder of unknown aetiology which is characterised by increased heart weight, subendocardial nodules, and the presence of histiocytoid cells within the myocardium. Involvement of the valves and conduction system is occasionally seen. The histiocytoid cells have decreased bundles of myofibrils and increased numbers of dilated mitochondria. ${ }^{1}$

Histiocytoid cardiomyopathy usually affects girls between the ages of 6 months and 2 years. However, cases have been reported as early as 3 days old and as late as 4 years old. Arrhythmias, including ventricular tachycardia, ventricular fibrillation, supraventricular tachycardia, and sudden death are common presentations. ${ }^{2}$ In the few cases of histiocytoid cardiomyopathy that have been diagnosed ante mortem, one patient benefited from treatment with amiodarone, ${ }^{3}$ and three others from surgical excision of the abnormal tissue. ${ }^{4}$ 

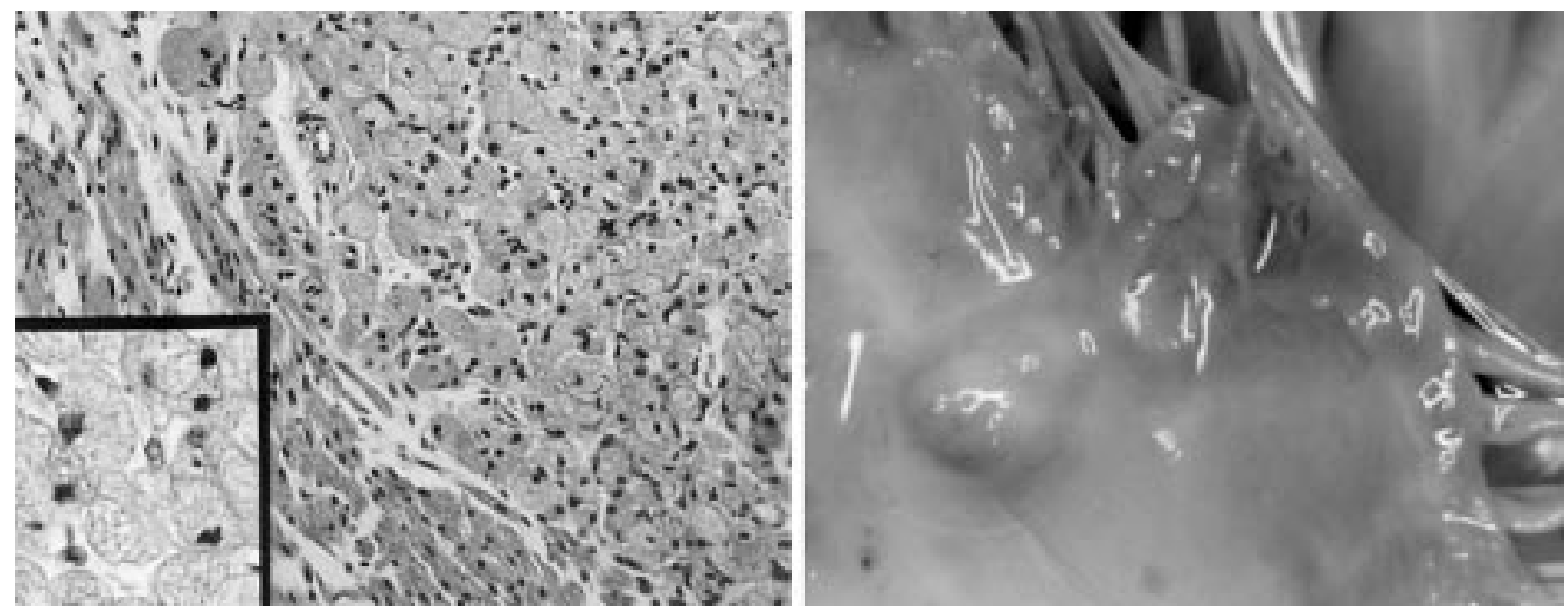

Figure 1 (Left) Histiocytoid cells within the myocardium showing large round myocytes with smooth borders, granular and eosinophilic cytoplasm, and small irregular nuclei. (Right) Subendocardial nodule $(0.5 \mathrm{~cm})$ on the atrial aspect of the tricuspid valve.

Although not as common as ventricular tachycardia, WPW is frequently found in patients with histiocytoid cardiomyopathy. ${ }^{5}$ While WPW has a generally favourable prognosis in children, cases of WPW in combination with histiocytoid cardiomyopathy are uniformly fatal, due to the poor prognosis of histiocytoid cardiomyopathy alone.

This research was supported in part by the Robert Wood Johnson Foundation.
1 Malhotra V, Ferrans VJ, Virmani R. Infantile histiocytoid cardiomyopathy: three cases and literature review. $\mathrm{Am}$ Heart $\mathcal{F}$ 1994;128:1009-21.

2 Prahlow JA, Teot LA. Histiocytoid cardiomyopathy: case report and literature review. F Forensic Sci 1993;38:142735

3 Gelb AB, van Meter SH, Billingham ME, et al. Infantile histiocytoid cardiomyopathy-myocardial or conduction system hamartoma: what is the cell type involved? Hum Pathol 1993;24:1225-31.

4 Garson A, Gillette PC, Titus JL, et al. Surgical treatment of ventricular tachycardia in infants. $N$ Engl F Med 1984;310; 1443-5.

5 Radford DJ, Chalk SM. Infantile xanthomatous cardiomyopathy. Aust Paediatr F 1980;16:123-5.

\title{
Ventricular and atrial septal defects, and right aortic arch associated with isolation of the left innominate artery from the aorta
}

\author{
A Gamillscheg, J I Stein, A Beitzke
}

\section{Department of \\ Pediatric Cardiology, Children's Hospital, \\ University of Graz, \\ Auenbruggerplatz 30, \\ A-8036 Graz, Austria \\ A Gamillscheg \\ J I Stein \\ A Beitzke}

Correspondence to:

Dr Gamillscheg

Accepted for publication 19 July 1999
A 6 month old boy with trisomy 21 was referred for preoperative cardiac catheterisation of a ventricular septal defect, an ostium secundum atrial septal defect, a right aortic arch, and a patent ductus arteriosus. On physical examination there was no cyanosis, a grade $3 / 6$ systolic murmur was heard at the left sternal border with a loud single second sound. Blood pressure on the right and left arm was $80 / 50 \mathrm{~mm} \mathrm{Hg}$.

Cardiac catheterisation revealed a left to right shunt with a Qp:Qs of 2.3:1 and a raised $\mathrm{Rp} / \mathrm{Rs}$ of 0.27 , as well as systemic blood pressure in the right ventricle and pulmonary arteries. A left ventriculography and an aortogram demonstrated a right aortic arch with only two branches arising from it, the first being the right common carotid artery followed by the right subclavian artery (fig 1). A small collateral vessel originated from the right sided descending aorta and extended obliquely upward to the left with a late slight opacification of the left subclavian artery that communicated through a left patent ductus arteriosus with the pulmonary artery. A right ventriculography and selective pulmonary angiography showed a left patent ductus arteriosus filling a left innominate artery and eventually the left common carotid and left subclavian arteries (fig 2).

The patient underwent patch closure of the ventricular septal defect and direct closure of the atrial septal defect via a right atriotomy. The left patent ductus arteriosus was divided and the left innominate artery was anastomosed with the ascending aorta by interposition of 


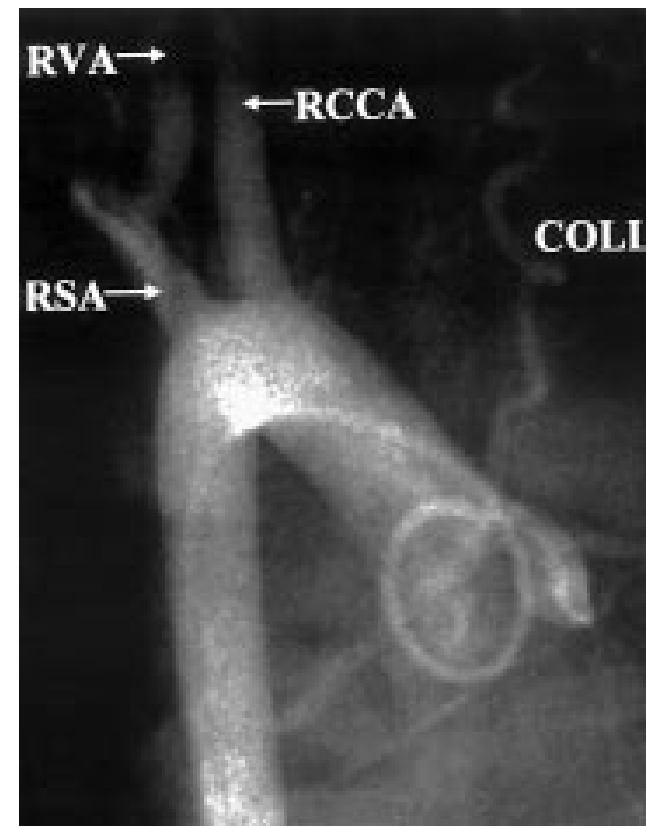

Figure 1 Aortogram (anterior posterior view): right aortic arch with right common carotid artery, right subclavian artery, and a collateral vessel originating from the descending aorta. No opacification of a left innominate artery. RCCA, right common carotid artery; $R S A$, right subclavian artery; $R V A$, right vertebral artery; $C O L L$, collateral vessel.

an $8 \mathrm{~mm}$ polytetrafluoroethylene tube. At follow up six months later the patient was asymptomatic with identical blood pressures on both arms.

\section{Discussion}

Right aortic arch with isolation of the left innominate artery is a very rare congenital anomaly in which the innominate artery loses its attachment to the aorta and is connected to the pulmonary artery via a left ductus arteriosus. ${ }^{1-4}$ The embryological development of this anomaly can be explained by interruption at two levels in the hypothetical double aortic arch model proposed by Edwards ${ }^{5}$ : one in the left posterior arch distal to the left ductus arteriosus and the other in the left anterior arch proximal to the left common carotid artery. ${ }^{4}$ Bilateral ductus arteriosus and atresia of the innominate artery or atresia of the left common carotid and subclavian arteries may be present. ${ }^{4}$ Usually the blood supply to the isolated arteries is provided via mediastinal collaterals and retrogradely via the circle of Willis and the left vertebral and carotid arteries. $^{23}$ The haemodynamic consequences depend on whether the left sided ductus arteriosus is patent or closed and, to some extent, on associated intracardiac anomalies. A closed ductus arteriosus predisposes to a congenital left subclavian steal phenomenon. ${ }^{2}$ A patent ductus arteriosus may lead to a

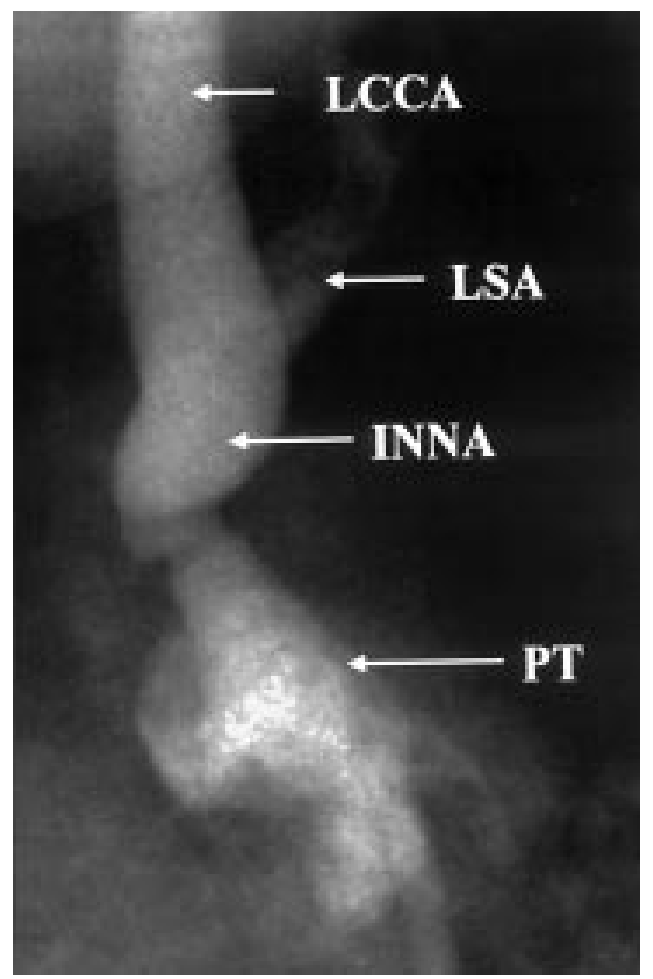

Figure 2 Pulmonary angiogram (sitting up view): opacification of a left innominate artery, a left common carotid artery, and left subclavian artery via a left patent ductus arteriosus. PT, pulmonary trunk; INNA, innominate artery; LSA, left subclavian artery; LCCA, left common carotid artery.

pulmonary steal phenomenon from the carotid and vertebral arteries causing an extracardiac left to right shunt. ${ }^{3}$ Clinically, the left radial and carotid pulses are weak and the blood pressure in the left arm is diminished. ${ }^{4}$ Symptoms of cerebral ischaemia have also been described. ${ }^{2}$

In our patient, systemic blood pressure in the pulmonary arteries prevented a significant pulmonary steal phenomenon via the patent ductus arteriosus and symptoms of reduced perfusion in the left arm. Aortography provides clear identification of these abnormal vascular structures. Usually the isolated arteries are opacified in late films via collateral pathways. ${ }^{3}$ Direct opacification of the left innominate artery via a patent ductus arteriosus during pulmonary angiography, as seen in our patient, results from increased pulmonary vascular resistance and pressure.

1 Fong LV, Venables AW. Isolation of the left common carotid or left innominate artery. Br Heart $\mathcal{F}$ 1987;57:552-4.

2 Garti IJ, Aygen MM. Right aortic arch and isolation of the left innominate artery from the aorta. Cardiovasc Intervent Radiol 1982;5:235-7

3 Martin EC, Mesko ZG, Griepp RB, et al. Isolation of the left innominate artery, a right arch, and a left patent ductus arteriosus. AfR Am f Roentgenol 1979;132:833-5.

4 Moes FCA, Freedom RM. Rare types of aortic arch anomalies. Pediatr Cardiol 1993;14:93-101.

5 Edwards JE. Anomalies of the derivatives of the aortic arch system. Med Clin North Am 1948;32:925-49. 


\title{
Sudden death and regional left ventricular fibrosis with fibromuscular dysplasia of small intramyocardial coronary arteries
}

\author{
A H S Lee, P B Gray, P J Gallagher
}

A 24 year old man collapsed, clutching his chest, while pushing a motor car. Resuscitation attempts were unsuccessful. He had previously lost consciousness while weightlifting. There was no history of drug abuse.

At postmortem examination the heart weighed $430 \mathrm{~g}$, normal for an $83 \mathrm{~kg}$ man. Cut section of the myocardium showed a $4 \mathrm{~cm}$ area of fibrosis in the lateral free wall of the left ventricle (fig 1). The epicardial coronary arteries had normal origin and no stenoses. The cardiac valves were normal. There was severe pulmonary oedema (right lung $800 \mathrm{~g}$, left lung $550 \mathrm{~g}$ ).

Histology showed that the area of fibrosis was associated with many abnormal small intramural arteries with thickening of the media, intima, or both (fig 2). Extensive sampling (more than 20 sections) of the ventricles and atria showed the myocardium and

\footnotetext{
Department of

Histopathology,

Southampton General

Hospital,

Southampton, UK

A H S Lee

P J Gallagher
}

Department of

Histopathology, Royal

Hospital, Chesterfield, UK

P B Gray

Correspondence to: Dr A H S Lee, Department of Histopathology, Nottingham City Hospital, Hucknall Road, Nottingham NG5 1PB, UK

Accepted for publication 16 August 1999

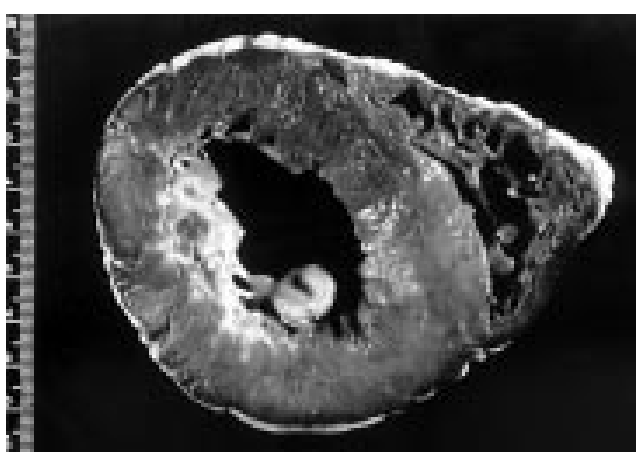

Figure 1 Cross section of both ventricles showing fibrosis in the lateral free wall of the left ventricle.

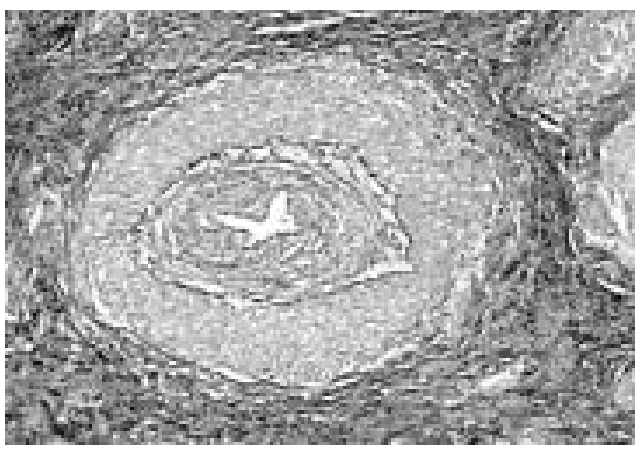

Figure 2 Section from the area of fibrosis showing vessel with notable intimal thickening (elastic van Gieson stain). small coronary vessels elsewhere were normal. No myocardial disarray was seen. The epicardial coronary arteries showed minimal intimal thickening. Vessels in the lung, kidney, spleen, and aorta were all normal.

Fibromuscular dysplasia is best recognised in the renal arteries and can affect the epicardial coronary arteries. Fibromuscular dysplasia of small coronary arteries has been described in several conditions: hypertrophic cardiomyopathy, Friedreich's ataxia, scleroderma, prolonged QT interval, Marfan's syndrome, progressive muscular dystrophy, tunnel aortic stenosis, mitral valve prolapse, and in the sinus node artery in sudden death. ${ }^{1-4}$ Many of these studies are based on small numbers and some have no control group. The best documented is hypertrophic cardiomyopathy in which such abnormal vessels are not only frequently seen ( $83 \%$ of hearts compared with $9 \%$ of controls) but also present in large numbers. ${ }^{3}$ The abnormal vessels are often seen in areas of fibrosis and have been identified in infants. It is likely that the fibrosis is secondary to (rather than a cause of) the abnormal vessels.

The striking feature of our case is the regional nature of the abnormality. Recently hamartoma of mature cardiac myocytes was described $^{5}$ in which discrete areas of hypertrophied disorganised myocytes were associated with thickened intramural vessels. The absence of myocardial disarray in our case is contrary to the diagnosis of hypertrophic cardiomyopathy. This pattern of regional fibrosis associated with fibromuscular dysplasia of intramyocardial arteries does not appear to have been described before. It seems likely that the fibrosis was secondary to the vascular abnormality; and the likely mechanism of sudden death was an arrhythmia. The regional nature of the abnormality raises the possibility of a congenital disorder, but there was no family history of heart disease, and investigation of several family members was negative. This case emphasises the importance of histology in myocardial fibrosis in the presence of normal epicardial coronary arteries.

This case was presented at the European School of Cardiovascular Pathology in the Academic Medical Centre, Amsterdam, Netherlands, October 1997. 
1 James TN. Morphologic characteristics and functional significance of focal fibromuscular dysplasia of small coronary arteries. Am 7 Cardiol 1990;65:12-22G.

2 Maron BJ, Redwood DR, Roberts WC, et al. Tunnel subaortic stenosis. Left ventricular outflow obstruction produced by fibromuscular tubular narrowing. Circulation 1976;54:404-16.

3 Maron BJ, Wolfson JK, Epstein SE, et al. Structural basis for myocardial ischemia in hypertrophic cardiomyopathy.
In: Virmani R, Forman MB, eds. Nonatherosclerotic ischemic heart disease. New York: Raven Press, 1989: ischemic

4 Burke AP, Farb A, Tang A, et al. Fibromuscular dysplasia of small coronary arteries and fibrosis in the basilar ventricular septum in mitral valve prolapse. Am Heart f 1997;134: 282-91.

5 Burke AP, Ribe JK, Bajaj AK, et al. Hamartoma of mature cardiac myocytes. Hum Pathol 1998;29:904-9.

\title{
SHORT CASES IN CARDIOLOGY
}

\section{Ventricular pre-excitation producing resolution of complete atrioventricular block}

\author{
C Wren
}

Department of

Freeman Hospital,

Newcastle upon Tyne

NE7 7DN, UK

C Wren

Correspondence to:

Dr Wren

Accepted for publication

6 August 1999
Paediatric Cardiology,

Coexistence of atrioventricular block and ventricular pre-excitation is rare. Most reported cases have been associated with structural heart disease and in many patients the atrioventricular (AV) block was incomplete. ${ }^{1-4}$

A 2 year old boy was referred for evaluation after a routine examination by his general practitioner had detected a slow pulse and a murmur. He was asymptomatic with normal growth and development. On examination he had a regular bradycardia of 65 beats/min and a grade 2 ejection murmur. The ECG showed
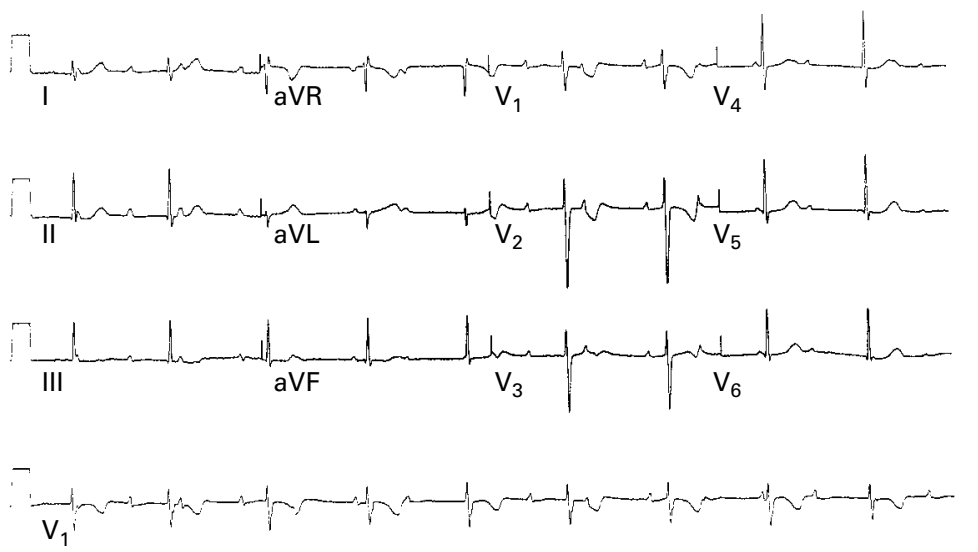

Figure 1 Complete atrioventricular block with normal QRS morphology.

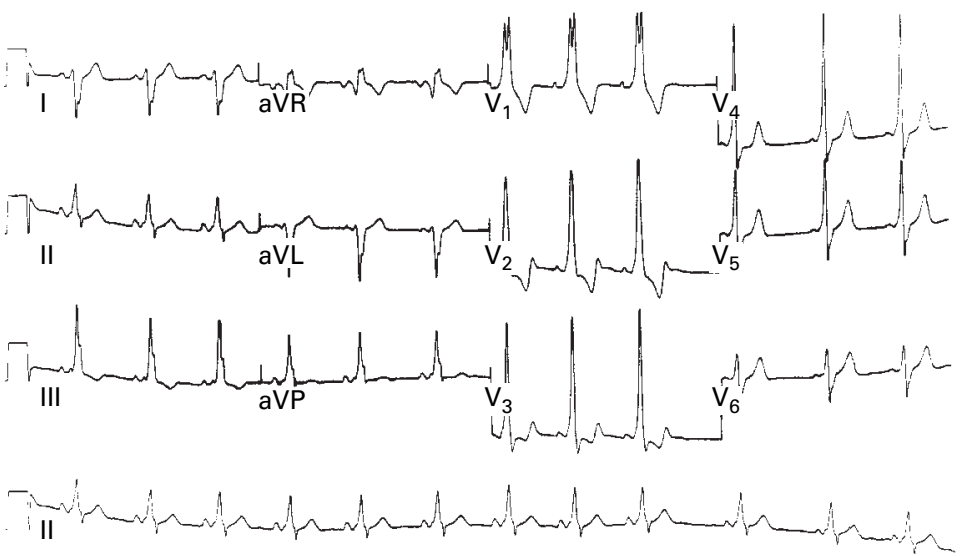

Figure 2 Sinus rhythm with 1:1 atrioventricular conduction and full ventricular pre-excitation. complete AV block with an atrial rate of 115 beats/min and a ventricular rate of 65 beats/ min (fig 1). The QRS duration and QT interval were normal. Echocardiography showed a structurally normal heart with normal ventricular function. A 24 hour ECG showed complete AV block throughout with an average ventricular rate of 50 beats $/ \mathrm{min}$ at night and 65 beats/min during the day. He remained well during follow up with no change in the clinical situation, ECG, or 24 hour ECG.

At 9 years old he attended for a routine clinic appointment. His parents reported an increase in his energy and he had taken up cross country running. On examination his pulse rate was 84 beats/min. His ECG showed sinus rhythm with 1:1 AV conduction and notable ventricular pre-excitation, with a pattern predicting a left posterolateral position for the accessory pathway (fig 2). A 24 hour ECG showed 1:1 AV conduction throughout with ventricular rates of up to 135 beats/min. A Bruce protocol exercise test showed sustained 1:1 conduction with pre-excitation throughout. He exercised for 15 minutes and achieved a maximum heart rate of 209 beats/min. He has been well during a further $3 \frac{1}{2}$ years' follow up with persisting 1:1 conduction on all investigations.

This case appears to be unique. The AV block was presumably congenital although maternal antibody testing was negative. The development of ventricular pre-excitation at 9 years old restored 1:1 conduction, and pathway conduction seems robust. Manifestation of ventricular pre-excitation during childhood occurs at an average of 8 years old, ${ }^{5}$ so this case is probably a fortunate coincidence. It provides the ideal solution to complete AV block.

1 Sigfússon G, Webber SA, Beerman LB. Coexistence of atrioventricular block and Wolff-Parkinson-White syndrome in children. Cardiol Young 1997;7:388-92.

2 Jordan JL, Yamaguchi I, Mandel WJ. Coexistence of the Wolff-Parkinson-White syndrome and atrioventricular nodal block. Chest 1978;74:79-82.

3 McLeod KA, Rankin AC, Houston AB. 1:1 atrioventricular conduction in congenital complete heart block. Heart 1998;80:525-6.

4 Timmis GC, Henke J, Gordon S, et al. The WolffParkinson-White syndrome in advanced atrioventricular block. Am 7 Cardiol 1971;28:592-7.

5 Perry JC, Garson A. Supraventricular tachycardia due to Wolff-Parkinson-White syndrome in children: early disappearance and late recurrence. $\mathcal{F}$ Am Coll Cardiol 1990;16: pearance 